\title{
Diseño del cuestionario de creencias referidas al consumo de alcohol para jóvenes universitarios
}

\author{
Design of the alcohol consumption beliefs \\ questionnaire for college students
}

\author{
Carolina Valencia Lara \\ Universidad Santo Tomás, Bogotá \\ Universidad Católica de Colombia, \\ Bogotá \\ Constanza Londoño Pérez ${ }^{* *}$ \\ Milena Amézquita \\ Javier Cortés \\ Mary Guerra \\ Andrés Hurtado Castiblanco \\ Jennifer Ordóñez \\ Universidad Católica de Colombia, \\ Bogotá \\ Recibido: 11 de febrero de 2009 \\ Revisado: 15 de abril de 2009 \\ Aceptado: 9 de mayo de 2009
}

\section{Resumen}

El objetivo del presente estudio fue diseñar y validar el Cuestionario de creencias referidas al consumo de alcohol para jóvenes universitarios. La prueba se aplicó a una muestra de 240 jóvenes universitarios entre los 16 y los 25 años, en la ciudad de Bogotá. El diseño del Cuestionario se basó en el Modelo de Creencias e incluyó los componentes del mismo: percepción de riesgo/vulnerabilidad/severidad, barreras percibidas, beneficios percibidos, normas subjetivas y claves para la acción. Los resultados muestran que la prueba tiene un buen nivel de validez y confiabilidad. Se presenta una descripción general de los resultados obtenidos con la muestra final de jóvenes. Finalmente, se discute sobre las aplicaciones y alcances del Cuestionario, y la necesidad de ampliar la muestra con jóvenes de otras ciudades.

Palabras clave: modelo de creencias, creencias sobre el alcohol, consumo de alcohol y jóvenes.

Artículo de investigación. Proyecto del Grupo de investigación: Psicología de la Salud.

Correspondencia: Constanza Londoño Pérez. Facultad de Psicología. Universidad Católica de Colombia. Correo electrónico: constanzalc@gmail.com. Dirección postal: Avenida Caracas No. 46 - 44 piso 1. Bogotá, Colombia. 


\section{Abstract}

The purpose of present study was to design and validate of Alcohol Beliefs Questionnaire in adolescents universitaries; this instrument was applicated to the nonrandom sample was conformed by 240 young's between the 16 and the 25 years university students at Bogotá city. The design of Questionnaire is based on the Health Belief Model and include theirs components: perceived risk and vulnerability, subjective norms, perceived barriers, perceived benefits and cues action. The results showed that the instrument has a good reliability and validity. Present description the results with sample include. Finally it is discussed the study limitations, for instance, althougth, it is recommended further applications of the instrument with other groups.

Key words: Health Belief Model, alcohol belief, alcohol comsuptions and youngs.

\section{Introducción}

El consumo de sustancias psicoactivas (SPA) en jóvenes es una de las problemáticas de salud pública que más se ha incrementado en los últimos años, especialmente por los efectos agudos que tiene sobre la salud, ya que puede producir enfermedades hepáticas, pancreatitis, gastritis agudas, crónicas atróficas, trastornos cardiovasculares y dos tipos de síndromes orgánico-cerebrales por causa del envenenamiento del cerebro por el exceso de alcohol.

También se asocia a otros problemas derivados, como embarazos no deseados, transmisión de infecciones por intercambio sexual, accidentes de tránsito y conducta delictiva. Adicionalmente, el problema del consumo se da cada vez a más temprana edad. En relación con esto, el Sistema de Vigilancia Epidemiológica para Uso Indebido de Sustancias Psicoactivas (VESPA) indica que en Bogotá el $37.7 \%$ de los casos de abuso de SPA corresponden a menores de 18 años, siendo los varones jóvenes entre los 16 y 20 años en quienes más se concentra la presencia de los casos (VESPA, 2007; Boletín Epidemiológico del Distrito, 2007).

En el caso específico del consumo de alcohol en adolescentes y jóvenes las cifras no son menos preocupantes. De una parte, éstos adoptan las prácticas de consumo de alcohol como una forma de expresar la autonomía e independencia propias de la vida adulta y, de otra, la ingesta de alcohol constituye una de las prácticas culturales que han hecho propias los universitarios. Según un estudio realizado por la Secretaria de Salud (VESPA, 2007) en Bogotá, durante el primer semestre de 2007, en 712 jóvenes, la principal sustancia de inicio en los casos de abuso registrados fue el alcohol, lo que equivale al $35.6 \%$ del total de los casos. Cabe anotar que entre los principales factores de riesgo de desarrollar una adicción se encuentran el inicio temprano y la escalada en el uso de sustancias, entre otros.

El consumo de alcohol en jóvenes, como fenómeno, resulta bastante complejo de predecir debido a la multiplicidad de factores asociados al mismo, entre ellos los estados afectivos, la motivación, los procesos conductuales de ingesta, el balance decisional (Flórez-Alarcón, Botero \& Moreno, 2005; Flórez-Alarcón, 2007), el género, la edad, el nivel socio-económico, los antecedentes familiares de ingesta, el patrón de consumo de pares y amigos (Rhodes \& Jason, 1990; Carmona \& Chávez, 1991; Donaldson, Graham, Piccinin \& Hansen (1995); Stice, Myers \& Brown, 1998; Ouellette, Gerrard, Gibbons \& Reis-Bergan, 1999; James, 2003; Guerrero, 2003; Uruk \& Demir, 2003; Hawkins, Cummins \& Marlatt, 2004; Londoño, 2007; Londoño, Valencia, Hernández \& León, 2007), la capacidad para resistir la presión del grupo dirigida a promover el consumo, el rendimiento escolar y la migración del país (Carvajal \& Andrade, 2005).

Dentro de esta multiplicidad de factores, el sistema de creencias que posee el individuo ha sido uno de los que ha demostrado tener mayor valor predictivo, en general, sobre la aparición de la conducta 
saludable y, de manera particular, sobre la de ingesta de alcohol, ya que el significativo componente social de las mismas, dado por su adquisición en el intercambio entre el sujeto y su cultura, facilita la aparición de conductas de corte social entre las que se cuenta el consumo de alcohol.

Rosentock (1974, citado por Sheeran \& Abraham, 1996) propuso el Modelo de Creencias en Salud (MCS) para explicar y predecir la aparición del comportamiento saludable, considerado como la resultante de una función interactiva entre diversos tipos de creencias que las personas tienen acerca de los eventos en salud. El MCS, considerado uno de los más influyentes en el estudio e intervención de los comportamientos individuales, relaciona teorías psicológicas de toma de decisiones para explicar la acción humana frente a situaciones de elección de comportamiento saludable. De acuerdo con este Modelo, las personas van tejiendo un armazón de afirmaciones explicativas sobre el mundo y los eventos que allí ocurren, tejido que ha sido definido como el grupo de creencias que el sujeto tiene acerca de la salud en general y específicamente sobre cada uno de los comportamientos como fumar, alimentarse y, para este caso, beber; creencias que definen directamente la actitud hacia ciertas prácticas en salud y de manera indirecta la aparición del comportamiento (Sheeran \& Abraham, 1996; Jackson \& Aiken, 2000).

De acuerdo con Cabrera, Tascón y Lucumi (2001), Becoña (1999), Grube y Agostinelli (1999) y Smith Klohn y Rogers (1991), en el MCS se plantea que la probabilidad de emitir una conducta saludable depende en gran medida del riesgo percibido, definido como la visión general que tiene la persona sobre el riesgo de otros similares de padecer una enfermedad; la vulnerabilidad percibida, entendida como la visión de los factores individuales que potencian el riesgo personal de sufrirla; y la severidad del riesgo, entendida como la gravedad de los daños físicos y funcionales derivados de la enfermedad. Además, se plantea que la existencia de percepciones relacionadas con la adopción de la conducta en términos de barreras (costos, conveniencia, dolor, incomodidad) para alcanzar la conducta saludable y los beneficios percibidos derivados de la conducta en la reducción del riesgo, tienen un valor predictivo importante.

Otro de los elementos cognitivos incluidos en el MCS son las claves para la acción, que hacen referencia a la existencia de una señal que desencadena la motivación para emitir la conducta, evoca las creencias relacionadas con la misma y permite la formación de la actitud necesaria para programarla (Rosentock, Strecher \& Becker, 1994), dichas claves pueden ser internas (síntomas físicos o percepciones corporales) o externas (recomendaciones de los medios de comunicación, recordatorios de los servicios de salud, consejos de los amigos, entre otros). La intensidad de una de estas claves para desencadenar la conducta puede variar de un individuo a otro, incluso en el mismo sujeto dependiendo de algunas variables intrínsecas.

Adicionalmente, se plantea la existencia de normas subjetivas como otro componente del MCS, éstas corresponden a la percepción que tiene el sujeto acerca de lo que los otros similares esperan que hagan, respecto de la conducta frente a su nivel de motivación para complacerlos, así, la norma subjetiva recoge la presión social que las personas perciben para emitir una conducta en particular, sumada a la motivación para ajustarse a dichas expectativas, este componente social plantea la importancia determinante y la función que el grupo social tiene sobre la adquisición de la conducta saludable (Moreno San Pedro, Gil Rosales-Nieto \& López Ríos, 2003; Moreno San Pedro, Gil Rosales-Nieto \& López Ríos, 2000; Londoño, 2007; Latimer, Floyd, Kariis, Novotna, Exnerova \& O`Brien, 2004).

En suma, para que la persona emita la conducta saludable necesita tener diversos tipos de creencias: 1) que es susceptible de sufrir la enfermedad, 2) que la ocurrencia de la enfermedad puede tener una severidad importante en su vida, y 3) que tomar una acción factible y eficaz puede ser benéfico para reducir la susceptibilidad y severidad de la enfermedad, y que estos beneficios superan en importancia y alcance a las barreras psicológicas percibidas respecto de la emisión de la acción preventiva. 
En particular, respecto del consumo de alcohol, Mora-Ríos y Natera (2001), Londoño, (2004; 2007), Londoño, García, Valencia \& Vinaccia (2005) y D'amico (2005) plantean que las creencias que las personas tienen acerca de su salud determinan la adquisición del hábito de ingesta alcohólica. En este sentido, la percepción de riesgo personal de abusar del alcohol, las expectativas acerca del consumo, las consecuencias percibidas del consumo, la actitud hacia el riesgo y las normas sociales percibidas, han sido identificadas como determinantes del éxito del MCS para reducir el abuso de sustancias en jóvenes.

Son numerosos los estudios que analizan el papel facilitador de las expectativas positivas en la conducta de consumo de alcohol, en ellos se reconfirma que la visión de efectos positivos (aparición de sensaciones agradables, aumento de habilidad social, facilitación de conducta y potenciación del desempeño sexual) provocan un incremento en la frecuencia de consumo (Stacy, Widaman \& Marlatt, 1990; Sheeran \& Abraham, 1996; Scheier \& Botvin, 1997; Grube \& Agostinelli, 1999; Turrisi \& Wiersma, 1999; Turrisi, Wiersma \& Hughes, 2000; Mora-Ríos \& Natera, 2001; Mora-Rios, Natera, Villatoro \& Villalvazo, 2002; Colder, Campbell, Ruel, Richardson \& Flay, 2002).

Mora-Ríos y Natera (2001) analizaron las expectativas típicas de jóvenes mexicanos a través de la aplicación del Cuestionario de Expectativas AEQ elaborado por Brown et al. (1980, citados por Mora-Ríos et al., 2000) y traducido al español por Mora-Ríos et al. (2002). En el estudio se encuentra que algunas expectativas están más referidas al género y a la construcción cultural del mismo. La imagen de fuerza y protección, la función activa en el acercamiento a la mujer y el cortejo son predominantemente masculinos. Asimismo, se observa que las creencias asociadas al desempeño sexual, a la agresividad y a sentimientos de poder se presentan principalmente en hombres. Se encontró relación significativa entre la expresividad verbal, la desinhibición, el incremento de la sexualidad, la interacción grupal, la reducción de la tensión psicológica, el incremento de la agresividad y sentimientos de poder con el patrón de consumo.

Los jóvenes con alto consumo obtuvieron las puntuaciones más elevadas en general en el AEQ, y sus principales expectativas fueron que el alcohol funcionaba como facilitador de la interacción grupal, como reductor de la tensión psicológica y como agente que incrementa la agresión y los sentimientos de poder. Al considerar las diferencias de acuerdo con el género, los hombres tuvieron mayores expectativas en cuanto a que el consumo de alcohol incrementaba su desempeño sexual, que las mujeres; también se observa que los hombres reportan más problemas asociados al consumo que las mujeres.

Por su parte, Arrivillaga, Salazar y Correa (2003) estudiaron en jóvenes universitarios colombianos el papel de las creencias sobre los comportamientos saludables y hallaron que éstos percibían el consumo de alcohol como favorable en ambientes de fiesta ya que facilitaba la diversión y los desinhibía. En esta misma línea, Londoño et al. (2005) adaptan el AEQ para población colombiana y encuentran que los adolescentes en este país asumen que consumir alcohol tiene beneficios, entre ellos, facilitar la interacción social, favorecer la expresividad verbal, desinhibir la conducta y reducir la tensión psicológica, resultados confirmados por Londoño (2007) en un estudio posterior, en el que, adicionalmente, se identifica la limitación del cuestionario, pues éste recoge únicamente las expectativas acerca de los efectos del consumo sin abordar otros elementos cognitivos descritos en el MCS y, por tanto, obvia aspectos cognitivos que han demostrado ser determinantes de la conducta de ingesta de bebidas alcohólicas, por lo cual es indispensable desarrollar un instrumento más general que englobe todos los elementos del modelo para poder así mejorar la comprensión del fenómeno y acercarse más a la posible predicción de este comportamiento de riesgo frecuente en la población universitaria. 


\section{Método}

\section{Participantes}

Los instrumentos se aplicaron a 240 estudiantes universitarios de dos instituciones privadas de la ciudad de Bogotá, que cursaban entre $4^{\circ}$ y 11 semestre en las jornadas diurna y nocturna, cuyas edades estaban comprendidas entre 18 y 34 años (media de 22.2); de los 240 participantes, 100 eran hombres y 140 mujeres. La muestra de conveniencia fue equilibrada incluyendo jóvenes clasificados en los cuatro niveles de consumo de alcohol.

\section{Instrumentos}

En la primera fase se diseñó el "Cuestionario de Creencias Acerca del Consumo de Alcohol" (CCAC) a partir de los constructos propuestos en el Modelo de Creencias en Salud para evaluar las creencias sobre el consumo de alcohol en población universitaria. La prueba estaba constituida por sesenta ítems con opciones de respuesta en escala tipo likert agrupados en cinco factores (vulnerabilidad/riesgo, normas, claves, barreras y beneficios) en los que se recogían los componentes básicos del modelo de forma equilibrada.

Luego, dos jurados expertos conceptuaron que los indicadores comportamentales definidos en los ítems eran pertinentes, suficientes y permi- tían evaluar el constructo global, concepto en el cual hubo una coincidencia de $83 \%$. A esto se suman algunas sugerencias en la redacción de las preguntas relacionadas con vulnerabilidad/riesgo y severidad. Posteriormente, se realizó una aplicación piloto a un grupo de cien sujetos con el fin de determinar si la presentación de los ítems en términos de redacción y de opciones de respuesta ofrecidas eran adecuadas. En el cuestionario se incluyó una pregunta de opción de respuesta dicótoma y una sobre la frecuencia y cantidad de consumo.

\section{Resultados}

En la fase dos, una vez realizados los ajustes, se procedió a hacer la aplicación final del instrumento para determinar la estructura definitiva. Luego de hacer un análisis de confiabilidad de la escala total y también de confiabilidad si cada uno de los ítems era eliminado, de los sesenta ítems se tomaron aquéllos que mejor comportamiento psicométrico presentaban en términos de aportar estabilidad y confiabilidad a la prueba, se eliminaron selectivamente cuarenta ítems hasta alcanzar un alfha de Cronbach de 0.87 , considerado un nivel de confiabilidad adecuado para este tipo de instrumentos. Cabe anotar que la aplicación del instrumento fue realizada por los investigadores en sesiones grupales previa firma del consentimiento informado de los participantes.

Tabla 1. Conformación final de la prueba y confiabilidad si el ítem es eliminado

\begin{tabular}{lcccr}
\hline & $\begin{array}{c}\text { Media de la escala } \\
\text { si se elimina el elemento }\end{array}$ & $\begin{array}{c}\text { Varianza de la escala si } \\
\text { se elimina el elemento }\end{array}$ & $\begin{array}{c}\text { Correlación elemento- } \\
\text { total,corregida }\end{array}$ & $\begin{array}{c}\text { Alfa de Cronbach } \\
\text { si se elimina el elemento }\end{array}$ \\
\hline CMCS1 & 17,43 & 73,858 &, 340 &, 872 \\
CMCS2 & 17,41 & 72,329 &, 468 &, 866 \\
CMCS15 & 17,97 & 73,136 &, 509 &, 865 \\
CMCS16 & 17,88 & 70,856 &, 594 &, 862 \\
CMCS18 & 18,44 & 73,443 &, 608 &, 863 \\
CMCS19 & 18,39 & 73,735 &, 544 &, 865 \\
CMCS20 & 18,41 & 75,136 &, 391 &, 869 \\
CMCS23 & 17,97 & 74,416 &, 305 &, 873 \\
CMCS24 & 18,36 & 74,276 &, 476 &, 867 \\
CMCS26 & 17,19 & 76,660 &, 162 &, 879 \\
CMCS27 & 18,09 & 73,020 &, 532 &, 865 \\
\hline
\end{tabular}




\begin{tabular}{lcccr}
\hline & $\begin{array}{c}\text { Media de la escala } \\
\text { si se elimina el elemento }\end{array}$ & $\begin{array}{c}\text { Varianza de la escala si } \\
\text { se elimina el elemento }\end{array}$ & $\begin{array}{c}\text { Correlación elemento- } \\
\text { total,corregida }\end{array}$ & $\begin{array}{c}\text { Alfa de Cronbach } \\
\text { si se elimina el elemento }\end{array}$ \\
\hline CMCS29 & 18,57 & 75,211 &, 464 &, 867 \\
CMCS31 & 17,49 & 70,453 &, 504 &, 865 \\
CMCS32 & 17,91 & 71,250 &, 524 &, 864 \\
CMCS33 & 18,19 & 73,001 &, 521 &, 865 \\
CMCS34 & 17,74 & 70,268 &, 644 &, 860 \\
CMCS35 & 17,50 & 71,086 &, 525 &, 864 \\
CMCS45 & 18,09 & 71,949 &, 531 &, 864 \\
CMCS56 & 18,27 & 71,178 &, 611 &, 861 \\
CMCS57 & 17,94 & 72,866 &, 394 &, 870 \\
\hline
\end{tabular}

En la tercera fase se llevó a cabo un análisis de componentes con la rotación Varimax y se observó que, aunque el diseño de la prueba se hizo teniendo cinco componentes principales (habiendo eliminado uno en la fase anterior), éstos no constituyen factores independientes sino que existe un único factor general denominado Creencias.

El cuestionario final está compuesto por veinte ítems, distribuidos en los cuatro componentes del modelo de creencias de manera equitativa, así: a) cinco que evalúan la percepción de riesgo y vulnerabilidad (1-2-15-16-17), b) cinco que evalúan los beneficios percibidos del consumo (3-4-
5-18-19), c) cinco que identifican las claves para la acción (11-12-13-14-20), y d) cinco que evalúan las barreras percibidas para dejar de consumir (67-8-9-10). Se eliminó el factor severidad ya que la escala incluía efectos generales no percibidos como inmediatos por los adolescentes y, por tanto, no valorados.

El $67 \%$ de los estudiantes reportó consumir alcohol y el 33\% no hacerlo. Entre los consumidores, el $15 \%$ reporta haber alcanzado estado de embriaguez en la última semana; el 7,9\%, en los últimos 15 días; el $5,7 \%$ en el último mes, y $52,1 \%$ en los últimos 6 meses. El $8 \%$ de consumidores omiten esta pregunta.

Tabla 2. Descripción de la muestra y detalle de los componentes de las creencias

\begin{tabular}{|c|c|c|c|c|c|c|c|c|c|}
\hline \multirow[b]{2}{*}{ Género } & \multirow[b]{2}{*}{$f$} & \multirow[b]{2}{*}{$\%$} & \multirow[b]{2}{*}{ Creencias } & \multicolumn{2}{|c|}{ Alto } & \multicolumn{2}{|c|}{ Moderado } & \multicolumn{2}{|c|}{ Bajo } \\
\hline & & & & $f$ & $\%$ & $\mathrm{~F}$ & $\%$ & $f$ & $\%$ \\
\hline Hombre & 100 & 42 & Vulnerabilidad/riesgo & 51 & 21 & 60 & 24 & 129 & 55 \\
\hline \multirow[t]{4}{*}{ Mujer } & 140 & 58 & Barreras & 196 & 82 & 32 & 13 & 12 & 5 \\
\hline & & & Beneficios & 203 & 85 & 31 & 12 & 6 & 3 \\
\hline & & & Claves & 34 & 13 & 75 & 31 & 131 & 56 \\
\hline & & & Norma subjetiva & 118 & 43 & 55 & 23 & 67 & 28 \\
\hline Consumo & $f$ & $\%$ & & & & \multicolumn{2}{|c|}{ Embriaguez } & $f$ & $\%$ \\
\hline Sí & 161 & 67 & & & & Últim & & 22 & 15 \\
\hline \multirow[t]{3}{*}{ No } & 79 & 33 & & & & \multicolumn{2}{|c|}{ Últimos 15 días } & 12 & 7,9 \\
\hline & & & & & & \multicolumn{2}{|c|}{ Último mes } & 3 & 5,7 \\
\hline & & & & & & \multicolumn{2}{|c|}{$\begin{array}{l}\text { Últimos } 6 \\
\text { meses }\end{array}$} & 57 & 52,1 \\
\hline
\end{tabular}


El 55\% considera que el riesgo personal de abuso de la sustancia es bajo; el $24 \%$ lo ve como moderado, y sólo el $21 \%$ lo percibe como alto, esto sin importar su nivel de consumo. El $82 \%$ percibe altas barreras de tipo social para alcanzar la abstinencia o disminuir su consumo; el $13 \%$ considera que tiene un nivel moderado de barreras para abandonar el consumo, y tan sólo el $5 \%$ percibe que no existen o son muy pocas las barreras que le dificultarían bajar el consumo.

De otra parte, en el componente referido a beneficios percibidos del consumo, $85 \%$ de los jóvenes reportó percibir altos beneficios o razones a favor del consumo; el $12 \%$ percibe un nivel moderado de razones para consumir, y únicamente el $3 \%$ identi- fica pocas razones para ingerir alcohol. Respecto de las claves para la acción, es decir, las señales que los llevan a consumir, sólo el $13 \%$ identifica como altas las claves (disparadores) para consumir, el $31 \%$ percibe un nivel moderado de claves (disparadores) para consumir y la mayor parte del grupo, 56\%, percibe como baja la presencia de claves para consumir.

Adicionalmente, se evidencia que al menos el $43 \%$ tiene un nivel alto de normas subjetivas dirigidas hacia el consumo; el 23\% tienen un nivel moderado de normas a favor del consumo y tan sólo el $28 \%$ restante reportan un nivel bajo de normas relacionadas con razones sociales para el consumo de alcohol.

Tabla 3. Análisis de correlaciones entre las variables

\begin{tabular}{lccccc}
\hline & Vulnerabilidad/riesgo & Claves & Beneficios & Barreras & Normas \\
\hline Consumo & - & $.537^{* *}$ & $.405^{* *}$ & - & $.401^{*}$ \\
Embriaguez & $-.426^{*}$ & $.671^{*}$ & $.773^{* *}$ & $.45^{*}$ & - \\
\hline
\end{tabular}

** p: $0.01,{ }^{*}$ p: 0.05

\section{Discusión y conclusiones}

En el diseño original de la prueba se incluyeron cinco componentes principales, se define que cuatro de ellos parecen ser aplicables al caso específico de consumo de alcohol en adolescentes: la percepción de vulnerabilidad y riesgo, los beneficios percibidos del consumo, las barreras para dejar de consumir y las claves o disparadores del consumo.

Respecto de la severidad, en términos del daño y su saliencia, la mayor parte de los jóvenes no señalaron ninguna de las opciones posibles, al parecer porque ninguna de ellas parecía tener proximidad temporal o las que se presentan con mayor frecuencia (guayabo, burlas, trasbocar) no representan cambios permanentes. No obstante, es preciso ahondar en estudios posteriores al respecto.

A partir del análisis psicométrico se concluye que el instrumento cuenta con un nivel de confiabilidad moderado y que los componentes del modelo incluidos en él no constituyen factores independientes, por tanto su aplicación y calificación debe hacerse usando la prueba completa. Respecto de la independencia de los componentes, cabe anotar que el sistema de creencias de las personas es un entramado de afirmaciones que se soportan unas a otras y son utilizadas para explicar las causas y razones por las que ocurre un evento, para luego hacer un juicio de valor sobre el mismo, y que sólo por razones prácticas autores como Rosentock (1974) y Sheeran y Abraham (1996) las han agrupado y denominado de forma tal que podrían parecer fenómenos independientes.

Igualmente, se evidencia la aplicabilidad del instrumento para llevar a cabo análisis cualitativos dirigidos a la descripción detallada de la percepción de riesgo y vulnerabilidad, beneficios, barreras y claves para la acción; y que su uso puede extenderse tanto a estudios descriptivos como a procesos de seguimiento en intervenciones dirigidas a incrementar la percepción de riesgo y vulnerabilidad, y la minimización de los beneficios 
y maximización de las barreras del consumir de alcohol, todos con efectos directos sobre el aumento motivación y disponibilidad al cambio.

Tras los análisis descriptivos de los datos recolectados a través del CCCA se reconfirman hallazgos obtenidos en otros estudios acerca del papel facilitador de las creencias sobre el consumo, especialmente en lo que atañe a la ingesta de alcohol en adolescentes ya que esta población además de negar la existencia del riesgo/vulnerabilidad, posee un grupo fuerte de creencias centradas en los beneficios del consumo y sus efectos positivos sobre el intercambio social. Sumado a esto se encuentra la baja percepción de parte del joven de los riesgos derivados de su conducta ya que las consecuencias evidenciadas por los adultos como razones de no consumo aparecen como muy lejanas en el tiempo para ellos.

En conclusión, su baja percepción de vulnerabilidad y riesgo los lleva a mantener el consumo, tal como lo muestran otros autores en estudios previos (Londoño \& Vinaccia, 2007; Londoño, 2007; Planes et al., 2006; Londoño et al, 2005; Londoño \& Vinaccia, 2005; Londoño, 2004; Vaillo, Galdon \& Dura, 2004; Cabrera et al., 2001; Mora-Ríos \& Natera, 2001; Becoña, 1999; Jackson \& Aiken, 2000).

En la misma línea se encuentran la percepción de fuertes barreras que dificultan el cese o disminución del consumo de alcohol (Pintanel, Capdevila \& Niñerota, 2006), la mayor parte de ellas de corte social y centradas en la aceptación del grupo de pares de dicho comportamiento, hallazgos que van en la misma línea de los obtenidos por Londoño et al. (2007), Planes et al. (2006), Cabrera et al. (2001) y Adalbjanardottir (2001) respecto del efecto que puede ejercer el grupo de homólogos sobre el inicio, mantenimiento y agudización en la ingesta de bebidas alcohólicas.

Las claves para la acción en este caso representan fuertes motivadores del consumo, ya que actúan como señales implícitas en el ambiente o derivadas del intercambio social que gatillan el inicio de la ingesta. Al respecto, Londoño (2007) describió la situación, mostrando cómo las instituciones universitarias están cercadas por un sinnúmero de establecimientos dedicados a expender alcohol a los jóvenes que manejan parte de las señales disparadoras (aumentar el volumen de la música, efectuar promociones, facilitar el ingreso de las mujeres), y otras provenientes del grupo como las invitaciones directas, las invitaciones a través de frases claves (entonces qué..., qué hay pa'hacer, nos vamos a beber tiendas) recopiladas en estudios previos realizados con población similar (Informe Invierte en $\mathrm{Ti}, 2000$ ).

Como conclusiones se puede decir, en primer lugar, que se cuenta con un instrumento que incluye diversos componentes de las creencias en torno al consumo del alcohol, que puede ser usado con jóvenes y adolescentes en Colombia, ya sea con fines de tamizaje en programas de detección o con fines de seguimiento de avances en procesos de acompañamiento para disminución o abandono del consumo de esta sustancia legal.

En segundo lugar, se puede decir que, en general, los análisis reconfirman los hallazgos anteriores sobre la función facilitadora que las creencias tienen sobre el consumo de alcohol (Sheeran \& Abraham, 1996; Stacy et al., 1990; Scheier \& Botvin, 1997; Grube \& Agostinelli, 1999; Turrisi et al., 2000; Informe Invierte en Ti, 2000; Mora-Ríos \& Natera, 2001; Colder et al., 2002; Londoño, 2007). En especial, si las expectativas positivas sobre los efectos del consumo priman sobre las negativas (Arrivillaga et al., 2003), se percibe como difícil o innecesario el cambio, de forma tal que existen múltiples señales que motivan el consumo y el joven ve que su grupo de pares espera y desea que consuma.

\section{Referencias}

Adalbjanardottir. S. (2001). Use substances Perceived control of adolescents: longitudynal and concurrentes analysis. Psychology of Addictive Behaviors, 15 (1), 25-32.

Arrivillaga, M., Salazar, I. C. \& Correa, D., (2003). Creencias sobre la salud y su relación con las prácticas de riesgo o de protección en jóvenes universitarios. Colombia Médica, 34, 186-195. 
Becoña, E. I. (1999). Bases teóricas que sustentan los programas de prevención de drogas. Plan Nacional sobre Drogas, España.

Cabrera, G., Tascón, J. \& Lucumi, 0. (2001). Creencias en salud: historia, constructor y aportes del modelo. Revista Nacional Facultad de Salud Pública, 19 (1), 91-101.

Carmona, A. \& Chávez, R. (1991). Expectativas de efectos positivos del alcohol adquiridas en el ambiente temprano (alcohólicos y no alcohólicos). Avances en Psicología Clínica Latinoamericana, 9, 151-162.

Carvajal, L. \& Andrade, D (2005). La escuela básica en la prevención del consumo de alcohol y tabaco: retrato de una realidad. Revista Latinoamericana Enfermagen, 13, 21-38.

Colder C., Campbell, R, Ruel, E., Richardson, J.L. \& Flay, B. (2002). A finite mixture model of growth trajectories of adolescent alcohol use: Predictors and consequences. Journal of Consulting Psychology, 70 (4), 976-985.

Donaldson, S.L., Graham, I.W., Piccinin, A.N. \& Hansen, W.B. (1995). Resistance- skills training and onset of alcohol use: Evidence know beneficial and potencially harmful effects in public schools and in private catholic schools. Health Psychology, 14 (4), 291-300.

Flórez-Alarcón, L. (2007). Psicología social de la salud: promoción y prevención. Colombia: Manual Moderno.

Flórez-Alarcón, L., Botero, M.M. \& Moreno, B. (Eds) (2005). Psicología de la salud: temas actuales de investigación en Latinoamérica. Colombia: Alapsa-Uninorte.

Guerrero, L. (2003). Por qué los jóvenes inician el consumo de licor antes de los 12 años. Crisol, Ciencia y Tecnología, 4 (159), 12-21.

Grube, J.W. \& Agostinelli, G.E. (1999). Perceived consequences and adolescent drinking. Nonlinear and interactive models of alcohol expectancies. Psychology of Addictive Behaviors, 13 (4), 303-312.

Hawkins, E.H., Cummins, L.H. \& Marlatt, G. A. (2004). Preventing substance abuse in american indian and Alaska native youth: Promising strategies for healthier communities. Psychological Bulletin, 130 (2), 304-323.

Informe Invierte en $\mathrm{Ti},(2000)$. Creencias sobre el consumo de alcohol en los adolescentes. Fondo de prevención Vial y Pontificia Universidad Javeriana, Bogotá.

Jackson, K.M. \& Aiken, L.S. (2000). A Psychosocial model of sun protection and sunbathing o young women: the impact of health beliefs, attitudes, norms, and self efficacy for sun protection. Health Psychology, 19 (5), 469478.

James. H. (2003). Individualización, pares y consumo de alcohol en adolescentes. Diario de Consulta y de Psicología Clínica Universidad de Baylor, 71 (3), 553-564.

Latimer, W., Floyd, Li., Kariis, T., Novotna, G., Exnerova, P. \& O’Brien, M. (2004). Peer and sibling substance use: predictors of substance use among adolescents in México. Revista Panamericana de Salud Pública, 15 (4), 225-232.

Londoño, C., García, W., Valencia, S.C. \& Vinaccia, S. (2005). Expectativas frente al consumo de alcohol en jóvenes universitarios. Anales de Psicología, 21 (2), 259-267.

Londoño, C. \& Vinaccia, S. (2005). Prevención del abuso en el consumo de alcohol en jóvenes universitarios: Lineamientos en el diseño de programas costo-efectivos Psicología y Salud, 15 (2), 241-249.

Londoño, C. \& Vinaccia, S. (2007). Actualización 2007: Prevención del abuso en el consumo de alcohol en jóvenes universitarios: lineamientos en el diseño de programas costo-efectivos AVISPA. Trabajo presentado en Congreso Pre- 
vención Basada en la Evidencia: Comunidades y droga, Bogotá.

Londoño, C., Valencia, S.C., Hernández, V. \& León, L. (2007). Diseño del Cuestionario de Resistencia a la Presión de Grupo en el consumo de alcohol. Suma Psicológica, 14 (2), 271-288.

Londoño, C. (2007). Construcción del modelo cognitivo social integrado para la prevención del consumo de alcohol en adolescentes universitarios. Típica, Boletín Electrónico de Salud Escolar, 3 (2). Recuperado el 30 de junio en http/www.tipica.org.co.

Londoño, C. (2004). Modelos de prevención aplicados a la conducta de consumo en jóvenes y adolescentes. En Manual de Psicología Clínica y de la Salud Hospitalaria, (1), 158-201. España: Psicom Editores.

Mora-Rios, J. \& Natera, G. (2001). Expectativas, consumo de alcohol y problemas asociados en estudiantes universitarios. Salud Pública de México, 43 (2), 89-96.

Mora-Rios, J., Natera, G., Villatoro, J. \& Villalvazo, R. (2002). Validez factorial del Cuestionario. Expectativas hacia el consumo de alcohol (AEQ) en estudiantes universitarios. Psicología Conductual, 8 (2), 319-328.

Moreno San Pedro, E., Gil Rosales-Nieto, J. \& López Ríos, F. (2000). Formación de creencias de salud en preadolescentes, un estudio descriptivo. Trabajo presentado en XXX Congreso of the European Association for Behavioural and Cognitive Therapies, Granada.

Moreno San Pedro, E., Gil Rosales-Nieto, J. \& López Ríos, F. (2003). El Modelo de creencias de salud: revisión teórica, consideración crítica y propuesta alternativa. Internacional Journal of Psychology and Psychological Therapy, 3 (1), 91-109.

Ouellette, J.A., Gerrard, M., Gibbons, E.V. \& ReisBergan, M. (1999). Parents, peers, and pro- totypes. Antecedents of adolescent alcohol expectancies, alcohol consumption, and alcohol - related life prob1ems in rural youth. Psychology of Addictive Behaviors, 13 (3), 183-197.

Planes, M., Gómez, A.B., Gras, S., Font-Mayolas, M., Cunil, M., Aymerich, M. \& Soto, J. (2006). Cambios en las percepciones de riesgo frente al SIDA de los estudiantes universitarios durante la última década. Cuadernos de Medicina Psicosomática y Psiquiatría de Enlace, 77 (76), 39-45.

Pintanel, M.; Capdevila, J. \& Niñerota, M. (2006). Barreras percibidas y actividad física: el autoinforme de barreras para la práctica de ejercicio físico. Revista de Psicología del Deporte, 15 (1), 53-69.

Rhodes, J.E. \& Jason, L.A. (1990). A social stress model of substance abuse. Jourmal of Consulting and Clinical Psychology, 58 (4), 395401.

Rosentock, I.M., Strecher, V.J. \& Becker, H.M. (1994). The health belief model and VIII risk behavior change. En: R. Diclemente y J.L. Peterson. Preventing AIDS, Theories and methods of behavioral interventions, (pp. 5-24). New York: Plenum Press.

Rosenstock, I.M. (1974). Historical origins of the Health Belief Model. Health Education Monographs, 2, 328-335.

Scheier, L.M. \& Botvin G. J. (1997). Expectancies as Mediators of the Effects of Social Influences and Alcohol Knowledge on Adolescent Alcohol Use: A Prospective Analysis. Psychology of Addictive Behaviors, 11 (1), 48-64.

Secretaría de Salud de Bogotá. Boletín Epidemiológico del Distrito, BED, (2007). Sistema de vigilancia epidemiológica de consumo de sustancias en la capital. 5 (2), 5-54.

Sheeran. P. \& Abraham, Ch. (1996). The Health Behaviours Model, En: Predicting Health Be- 
haviours (pp. 23-70). Philadelphia: Norman y Conner.

Sistema de Vigilancia Epidemiológica en el consumo de sustancias psicoactivas VESPA. (2007). Abuso de sustancias psicoactivas en Bogotá D. C., enero a junio de 2007. Colombia.

Stice, E., Myers. M. \& Brown, S. (1998). A longitudinal grouping analysis of adolescent substance use escalation and desescalation. Psychology of Addictive Behaviors, 12 (1), 14-27.

Smith Klohn, L. \& Rogefs, R.W. (1991). Dimensions of severity of health threat: the persuasive effects of visibility, time of onset and rate of onset on young women's intentions lo prevent osteoporosis. Health Psychology, 10, 323 - 329.

Stacy, A. W., Widaman, K. F. \& Marlatt, G. A. (1990). Expectancy models of alcohol use. Psychology of Addictive Behaviors, 6 (2), 918-928.
Turrisi, R., Wiersma, K.A. \& Hughes, K.K. (2000). Binge-drinking- related consequences in college students: roles of drinking beliefs and mother-teen communications. Psychology Addictive Behaviors, 14 (4), 342-355.

Turrisi, R. \& Wiersma, K.A. (1999). Examination of judgments of drunkenness, binge drinking, and drunk driving tendencies in teens with and without a family history of alcohol abuse. Alcoholism: Clinical and Experimental Research, 23, 1-8.

Uruk, A. \& Demir, A. (2003). Pairs and family rol on prediction of adolescents level isolation. Joumal of Psychology, 137 (2), 98-114.

Vaillo, A., Galdon, J. \& Dura, M. (2004). Health Beliefs and Breast Cancer Screening Program Participation in Valencian Community, Revista Española de Salud Pública, 78 (1), 65-82. 